horn of hyoid to the midpoint of the submental space. The operating time was approximately 20 minutes. All patients went on to receive radical radiotherapy within 2 weeks of the procedure. Unstimulated pre-radiation and post-radiation salivary output was measured by cannulating the submandibular ducts.

The mean salivary output of the transferred gland was $73 \%$ after radiotherapy, compared with only $27 \%$ for the untransferred, ipsilateral gland. The authors conclude that SMSGT maintained adequate salivary gland function in these patients and that the procedure was simple, safe and cost effective.

Original article Pathak KA et al. (2004) Upfront submandibular salivary gland transfer in pharyngeal cancers. Oral Oncol 40: 960-963

\section{Improved long-term survival in ovarian cancer}

Since cure is unlikely in most patients with recurrent ovarian cancer, treatments are aimed at prolonging survival while maintaining quality of life. A trend towards improved survival has been demonstrated in patients treated with pegylated liposomal doxorubicin compared with topotecan. Gordon et al. have recently completed their analysis of long-term survival data from the same study.

The analysis covered 474 women with epithelial ovarian cancer who either failed to respond to first-line, platinum-based chemotherapy or recurred after treatment. Patients subsequently randomized to pegylated liposomal doxorubicin $(n=239)$ had significantly better survival than those treated with topotecan ( $n=235)$. Median survival was 62.7 and 59.7 weeks for the two groups, respectively. This corresponded to an $18 \%$ reduction in the risk of death in the pegylated liposomal doxorubicin group. Patients with platinum-sensitive disease (i.e. those who responded to initial treatment and were then progression-free for >6 months) were analyzed separately. These women showed a more pronounced survival benefit when treated with pegylated liposomal doxorubicin, with a $30 \%$ reduction in the risk of death compared with those in the topotecan group. For patients with platinum-refractory disease, there was no significant difference in survival between the two treatment groups.
In conclusion, pegylated liposomal doxorubicin significantly prolonged survival in this long-term study, compared with topotecan. The authors suggest that the drug should be treatment of choice for patients with relapsed ovarian cancer.

Original article Gordon AN et al. (2004) Long-term survival advantage for women treated with pegylated liposomal doxorubicin compared with topotecan in a phase 3 randomized study of recurrent and refractory epithelial ovarian cancer. Gynecol Oncol 95: 1-8

\section{Omitting ALND in breast cancer: long-term results}

Axillary lymph node dissection (ALND) is now commonly omitted in sentinel node (SN)negative breast cancer patients. Although this approach avoids ALND-associated morbidity, its safety depends on the accuracy of the SN biopsy. Torrenga and colleagues have evaluated the long-term rate of axillary recurrences in patients treated in this way.

The prospective study included 104 SNnegative breast cancer patients. All patients had undergone SN biopsy by the triple technique (preoperative lymphoscintigraphy, intraoperative use of blue dye, and localization of the SN using a handheld gamma probe). Since H\&E staining and immunohistochemistry of the harvested SNs showed no tumor, no further ALND was performed. Patients were treated with breastconserving therapy or modified radical mastectomy, plus systemic therapy in some cases. The women were followed up every 3 months for the first year and 6-monthly thereafter.

During the follow-up period (median 57 months), only one local axillary recurrence was recorded. Three further patients developed distant metastases (in bone, brain and lung) and two of these patients died. The overall survival and disease-free survival rates were therefore $98 \%$ and $97 \%$, respectively, whereas the local control rate was $99 \%$.

The authors conclude that omitting ALND did not appear to have compromised local control or survival in these SN-negative patients, after more than 4 years' follow-up.

Original article Torrenga $\mathrm{H}$ et al. (2004) Omitting axillary lymph node dissection in sentinel node negative breast cancer patients is safe: a long term follow-up analysis. J Surg Oncol 88: 4-8

\section{GLOSSARY}

H\&E

Hematoxylin and eosin 